\title{
Thermal stability analysis of YBCO coated conductors subject to over-currents
}

\author{
E Martínez $^{1}$, L.A. Angurel ${ }^{1}$, J. Pelegrín $^{1}$, Y.Y. Xie ${ }^{2}$, V. Selvamanickam ${ }^{2}$ \\ ${ }^{1}$ Instituto de Ciencia de Materiales de Aragón, CSIC - Universidad de Zaragoza, C/ María \\ de Luna 3, 50018 Zaragoza, Spain \\ ${ }^{2}$ SuperPower Inc., Schenectady, NY 12304 USA
}

Keywords: quench, thermal stability, YBCO, superconducting coated conductors, over-currents

Classification numbers: 74.72.Bk, 74.25.Fy, 84.71.Mn

\begin{abstract}
:
The thermal stability of superconducting YBCO coated conductors subject to over-currents are analysed. We have studied the effect of DC and AC over-current pulses in $\mathrm{Cu}$-stabilized and non-stabilized coated conductors by measuring the electric field and temperature profiles of these conductors immersed in liquid nitrogen. Current pulses of short duration of about $90 \mathrm{~ms}$ and long duration of a few seconds were applied to the samples. Three different cooling regimes of the liquid nitrogen: convection, nucleate boiling and film boiling were observed, and their influence in the recovery time of superconductivity in the coated conductors after the over-current pulses has been analysed. We have studied the recovery behaviour under two different conditions, in which the current was set to zero and to the operating current after the current pulses. These experiments simulated the conditions during an over-current situation in different electric power applications with special attention given to the behaviour of these coated conductors acting as in fault current limiters.
\end{abstract}




\section{Introduction}

$\mathrm{YBa}_{2} \mathrm{Cu}_{3} \mathrm{O}_{7-\delta}(\mathrm{YBCO})$ coated conductors are the subject of intense research activities in recent years due to their promising properties for use in electric power applications, such as cables, magnets, motors, generators, superconducting fault current limiters (SFCL), etc. In most applications the nominal current is below the critical current, $I_{\mathrm{c}}$, and, therefore the dissipation in the superconducting material is only generated by the AC losses. On the contrary, SFCLs, especially the resistive type, are based on the superconductor-normal transition induced by a current higher than $I_{\mathrm{c}}$, although have also low dissipation at normal operation. In general, the analysis of thermal stability of superconducting composite materials is of great importance since heat disturbances and over-currents may occur during normal operation, which may cause the transition to the normal state of the overall conductor (quench), and may result in the irreversible damage of the material. This analysis, comprising the study of the superconducting-normal transition homogeneity along the conductor, as well as the thermal recovery after the quench, is crucial in the case of resistive SFCL.

From the point of view of thermal stability, most applications require the use of a stabilizer, which is generally a layer of metal with high thermal and electric conductivities, such as $\mathrm{Cu}, \mathrm{Ag}$ or $\mathrm{Au}$, in proximity of $\mathrm{YBCO}$. However, the use of a stabilizer may not be suitable for resistive SFCLs because the decrease of the conductor electric resistance in the normal state lowers the fault current limiting performance. In this case, the resistivity in the normal state can be increased by decreasing the thickness of the stabilizer [1] or by using a higher resistive alloy such as Ag-Au [2,3].

Thermal stability of a superconducting material is generally studied by applying over-currents $[1,3,4,5]$ or heat pulses $[6,7]$ to the conductor and analysing the electric field and temperature profiles along the conductor. These experiments simulate the behaviour of the superconducting conductor during a fault in different applications and allows the determination of parameters such as the minimum quench energy, quench propagation velocity, quench current, etc.

In this paper we analyse the effect of DC and $\mathrm{AC}$ over-currents in $\mathrm{Cu}$-stabilized and non-stabilized coated conductors. The electric field and the temperature of the sample immersed in liquid nitrogen and subject to over-currents are analysed for current 
pulses of short duration of about $90 \mathrm{~ms}$ and long duration of few seconds. The recovery times after the pulse are also analysed.

\section{Experimental}

The measurements were performed on coated conductors fabricated by SuperPower using Ion Beam Assisted Deposited MgO technology for buffer layers and metal organic chemical vapor deposition for YBCO. The main characteristics of the analysed samples are collected in table 1. The thickness of the hastelloy substrate and YBCO layer is $50 \mu \mathrm{m}$ and $1 \mu \mathrm{m}$, respectively. The width of sample S1 and S2 is $4 \mathrm{~mm}$. $\mathrm{S} 1$ is a Cu-stabilized sample, which has a $2 \mu \mathrm{m}$ Ag layer deposited on top of YBCO and then the entire conductor structure is stabilized by electroplating a $20 \mu \mathrm{m} \mathrm{Cu}$ layer on all sides. Sample S2 has $2 \mu \mathrm{m}$ Ag layer, but not copper. Sample S3 is similar to S2 except for the width, which is $2 \mathrm{~mm}$. The length of the samples is $8 \mathrm{~cm}$ plus $1 \mathrm{~cm}$ for each current contact. The end-to-end $I_{\mathrm{c}}$ of the samples at $77 \mathrm{~K}$ was 123,140 and $40 \mathrm{~A}$ for samples S1, S2 and S3, respectively. Homogeneity of $I_{\mathrm{c}}$ along the sample has also been tested and it has been observed that the differences in the critical current values between different $2-\mathrm{cm}$ regions of the sample is always lower than $1 \mathrm{~A}$. The electric resistivity and specific heat (C) of the samples have been measured using a Physical Property Measurement System (PPMS) from Quantum Design. The values at different temperatures are also collected in table 1. A rough estimation of the specific heat values of the samples can be also obtained assuming that $\mathrm{C}$ values of coated conductors are dominated by those of hastelloy and copper. The values obtained this way, using the values and procedure described in [6], are about 5-10\% and $10-25 \%$ higher than the measured ones (in the range between 80 and $300 \mathrm{~K}$ ) for the tapes with (S1) and without copper stabilizer (S2), respectively.

Time-resolved electric field measurements along the sample have been carried out by means of a Data Acquisition (DAQ) device. Four voltage taps were distributed uniformly on the sample to record the electric field evolution in different sample regions. The distance between voltage taps 1 and 4 and the current contacts is $1 \mathrm{~cm}$, and the distance between each two adjacent voltage taps is $2 \mathrm{~cm}$, approximately. The temperature was measured by three miniature type $\mathrm{K}$ thermocouples soldered directly to the sample for a fast response with reduced thermal mass and contact resistance and 
placed between the voltage taps. All the contacts are on the side of the tape opposite to the substrate and therefore closer to the YBCO layer. During the tests, the samples were surrounded by liquid nitrogen $\left(\mathrm{LN}_{2}\right)$ on both sides of the tape and they were placed with their longitudinal axis and their flat faces parallel and perpendicular to the liquid surface level, respectively. A given AC or DC current, higher than the critical current value, was applied to the sample for a period of time in the range of 4 seconds for long pulses and about $90 \mathrm{~ms}$ for short pulses. The AC current in the circuit was measured by a Rogowski coil, and the DC current by a calibrated resistance in series with the superconductor. The maximum available DC current is 800 A. For most experiments, the maximum AC current was 307 A (peak value), but recent changes in our experimental set-up have allowed us measuring up to $450 \mathrm{~A}$ (peak) in the latest experiments. In the paper, for AC experiments, $I_{\mathrm{p} \text {-short }}$ corresponds to the peak current in short-circuit, i.e. without the superconductor, and the peak current flowing through the circuit with the superconductor connected is called $I_{\mathrm{p}}$. The frequency of the AC current was $59 \mathrm{~Hz}$ in all cases, close to the frequency of the electric network but slightly different in order to minimize the noise coming from it.

\section{Results and discussion}

\subsection{Cu-stabilized tapes under DC over-currents}

The typical behaviour of the Cu-stabilized sample S1 under DC current pulses is shown in Figure 1. The DC current pulses of 140, 145 and 147 A correspond to reduced current values, $i=I / I_{\mathrm{c}} \sim 1.14,1.18$, and 1.19 , respectively. It must be noted that in this experimental configuration the pulse starts at $t=0$ and achieves a constant value at $t \sim$ $0.33 \mathrm{~s}$. It is observed that for $I=140 \mathrm{~A}$ the electric field remains constant $\left(\sim 44 \mu \mathrm{Vcm}^{-1}\right)$ during the time of the pulse, indicating temperature $(\sim 77 \mathrm{~K})$ is constant, which is consistent with the result shown in figure 1(b). For $I=145 \mathrm{~A}$, the electric field reaches a value of $85 \mu \mathrm{Vcm}^{-1}$ at the beginning of the pulse and increases up to $330 \mu \mathrm{Vcm}^{-1}$ at the end of the pulse, which implies that the power dissipated in the sample is higher than the nitrogen cooling capability, in agreement with the temperature increase observed in figure 1(b). When further increasing the current slightly to $147 \mathrm{~A}$, sudden increases in electric field and temperature are observed at $t \sim 2 \mathrm{~s}$. This so-called thermal runaway with $E$ up to $15 \mathrm{mVcm}^{-1}$ (corresponding to a power dissipation of $2.8 \mathrm{Wcm}^{-2}$ ) and $\Delta T=$ 
$T-77=8.2 \mathrm{~K}$ is immediately followed by sudden drop in both, electric field and temperature to $E=0.4 \mathrm{mVcm}^{-1}$ and $\Delta T=0.7 \mathrm{~K}$, respectively, and then both magnitudes remain almost constant for the rest of the current pulse. This sudden decrease in the electric field and temperature can be attributed to the improvement of the cooling capability of the nitrogen liquid when changing from natural convection to the much more effective nucleate boiling regime [8-10], and will be discussed in more detail later.

For clarity purposes, figure 1 only shows the temperature and electric field measured at the centre of the sample (i.e. measured between taps 2 and 3 and by the thermocouple placed between them). The measurements obtained from the three voltages and thermocouples are very similar in all cases, reflecting a very good homogeneity of the sample, in both aspects, the critical current and the stabilizer characteristics (resistance of the stabilizing layer and its adhesion to YBCO). For example, for $I=147 \mathrm{~A}$, the temperature drop measured by the three thermocouples occurs at times $t=2.65,2.94$ and $2.95 \mathrm{~s}$, respectively, and the measured temperatures are also very similar: $\Delta T=5,7$ and $8.5 \mathrm{~K}$, respectively, at the maximum level, and $\Delta T=0.5,0.7$ and $0.9 \mathrm{~K}$, respectively, after the temperature drop.

\subsection{Cu-stabilized tapes under AC over-currents}

The behaviour of the same sample (S1) subject to a long AC current pulse of peak value in short-circuit (i.e. without superconductor), $I_{\mathrm{p} \text {-short, }}$ above $I_{\mathrm{c}}$ is very similar to the previous DC case. The main difference is that the temperature and electric field jumps occur at higher currents, as shown in figure 2. The inset of figure 2(a) shows the detail of the electric field at the beginning of the pulse, where the typical non-sinusoidal shape of $E(t)$ for superconductors at $T<T_{\mathrm{c}}$ and $I_{\mathrm{p}}>I_{\mathrm{c}}$ is seen due to their non-linear $I-V$ characteristics. The detail of $E(t)$ at around $t=3 \mathrm{~s}$ is also shown in the lower inset, where the sudden decrease of $E(t)$ and the change from a sinusoidal (for $T>T_{\mathrm{c}}$ ) to a nonsinusoidal curve is observed. The temperature just before the drop is $\Delta T=6 \mathrm{~K}$ and after that, it remains constant at $\Delta T=0.7 \mathrm{~K}$, similarly to the DC case. As for the previous case, all voltage taps and thermocouples give very similar values. It must be noted that in this case there is not current limitation by the superconducting sample, being the maximum current $I_{\mathrm{p}}=I_{\mathrm{p} \text {-short }}=178 \mathrm{~A}\left(I_{\mathrm{p}} / I_{\mathrm{c}}=1.45\right)$ from $t=0$ until the end of the pulse. The average power dissipated in a cycle just before the temperature drop is in this case 2.2 $\mathrm{Wcm}^{-2}$, very similar to that observed previously for DC currents and also found in the 
literature for other systems $[10,11]$. For higher currents, $I_{\mathrm{p} \text {-short }}=183 \mathrm{~A}=1.49 I_{\mathrm{c}}$, the jump occurs sooner at $t \sim 1.5 \mathrm{~s}$, but the average power dissipated in a cycle just before the temperature drop is similar, $2.7 \mathrm{Wcm}^{-2}$.

Figure 3 shows the current, electric field and temperature increase of the same sample S1 subject to AC over-current pulses of short duration ( $90 \mathrm{~ms})$ and peak values in the absence of superconductor of $I_{\mathrm{p} \text {-short }}=235$ and $307 \mathrm{~A}$ and $450 \mathrm{~A}\left(I_{\mathrm{p} \text {-short }} / I_{\mathrm{c}}=1.9,2.5\right.$ and 3.7, respectively). In order to show the homogeneity of the electric field along the sample, the three electric fields measured between three pairs of adjacent voltage taps at two current pulses are shown in figures 3(a), 3(b) and 3(c), respectively, and they are hardly distinguishable in most cases as shown in the figures. In the case of $I_{\text {p-short }}=307$ $\mathrm{A}$ and $450 \mathrm{~A}$, the temperature increase during the pulse causes an increase of the electric field and a small decrease of the current during the pulse [by a factor about 0.95 and 0.83 at the end of the pulse, see figures 3(b) and 3(c), respectively]. This is unlike the case of $I_{\mathrm{p} \text {-short }}=235 \mathrm{~A}$, where the current remains constant during the pulse, and the temperature of the sample well below $T_{c}$, as reflected by the non-sinusoidal $E(t)$ curve.

Figure 3(d) shows the temperature measured by one of the thermocouples for the above over-currents. As it is observed in the figure,

i) when the temperature increases up to $\sim 5 \mathrm{~K}$ at the end of pulse, the temperature recovery is exponential (linear in log scale of the figure) down to $\Delta T=0.1-0.2 \mathrm{~K}$;

ii) the temperature recovers faster when the temperature of the sample increases up to the range $6-7 \mathrm{~K}$ than when the temperature of the sample is just 1-3 $\mathrm{K}$ above liquid nitrogen temperature. For example, the recovery time for $I_{\mathrm{p} \text {-short }}=235 \mathrm{~A}$ is longer than those for higher currents $307 \mathrm{~A}$.

iii) for short-circuit currents, $I_{\mathrm{p} \text {-short, }}$ higher than 420-450 A, the temperature increase of sample $\mathrm{S} 1$ during the pulse is considerably higher (up to $\Delta T=60 \mathrm{~K}$ ). In this case, the temperature recovery after the pulse is very slow down to $\Delta T \sim 20 \mathrm{~K}$, and suddenly becomes faster until $\Delta T \sim 1-2 \mathrm{~K}$.

A more detailed analysis of the observed behaviour will be done in section 3.4.

\subsection{Non-stabilized tapes under AC over-currents}

Figure 4 shows the current and electric field of the non-stabilized sample S2 subject to the similar short AC current pulses as in figure $3: I_{\mathrm{p} \text {-short }}=235$ and $307 \mathrm{~A}\left(I_{\mathrm{p}-}\right.$ 
short $/ I_{\mathrm{c}}=1.7$ and 2.2 , respectively) with duration of $\sim 90 \mathrm{~ms}$. The absence of the $\mathrm{Cu}-$ stabilizer results in a considerably higher value of $E(t)$ and higher temperature increase with respect to the observations in sample S1. For both current values, there is a current limitation effect due to the superconductor, which is reflected in the decrease of the current by a factor about 0.7 and 0.4 at the end of the pulse, for the cases of $I_{\text {p-short }}=235$ and 307A, respectively. It must be noted that although the temperature and electric field along the sample are not homogeneous at 235A, the transition to the normal state of the sample is very homogeneous at 307A and all three electric fields reach $E_{\text {peak }} \sim 0.4 \mathrm{Vcm}^{-}$ ${ }^{1}$ at the end of the pulse, as shown in figure 4(b).

The temperature measured at the centre of the sample S2 during different current pulses with duration of $\sim 90 \mathrm{~ms}$ is shown in figure 5 . The behaviour is similar to the observed for sample S1 of figure 4(d). The temperature recovers faster when the temperature of the sample increases up to the range 4-10 K than when the temperature of the sample is just $1-3 \mathrm{~K}$ above liquid nitrogen temperature. For $I_{\mathrm{p} \text {-short }}$, higher than $265 \mathrm{~A}$, the temperature increase during the pulse is considerably higher (up to $\Delta T=60$ $100 \mathrm{~K}$ ). In this case, the temperature recovery after the pulse is very slow down to $\Delta T \sim$ $30 \mathrm{~K}$, and suddenly becomes faster down to $\Delta T \sim 1 \mathrm{~K}$.

\subsection{Thermal recovery after over-current pulses. Heat transfer coefficient, $h$}

The temperature observations for samples S1 and S2 can be explained by analysing the heat balance equation of the system during recovery at zero current:

$$
A C(T) \frac{d T}{d t}=A \frac{d}{d x}\left(K(T) \frac{d T}{d x}\right)+P h \Delta T,
$$

where $x$ is the distance along the wire, $K$ is the longitudinal thermal conductivity of the coated conductors in $\left[\mathrm{Wm}^{-1} \mathrm{~K}^{-1}\right], C$ their specific heat per unit volume in $\left[\mathrm{JK}^{-1} \mathrm{~m}^{-3}\right], A$ their cross-sectional area in $\left[\mathrm{m}^{2}\right], P$ in $[\mathrm{m}]$, the perimeter per unit length in contact with the nitrogen, which in this case would be approximately twice the sample width $(P \sim$ $2 w), h$ the heat transfer coefficient in $\left[\mathrm{Wm}^{-2} \mathrm{~K}^{-1}\right]$, and $\Delta T$ the temperature difference between the sample and the liquid nitrogen. Here, we have considered that the temperature gradient across the different layers of the coated conductor can be neglected, although more work is needed to quantify this effect. Moreover, as a first approximation, if we assume a long conductor, the thermal conductivity term would be 
negligible compared to the cooling capability of the liquid (given by the second term in the right hand side of the equation) and therefore equation (1) can be approximated by:

$$
A C(T) \frac{d T}{d t} \approx P h \Delta T
$$

Since $T=\Delta T+77 \mathrm{~K}$, for the case of constant $C(T)$ would give exponential decays of the temperature:

$$
\Delta T \approx \Delta T_{o} \exp (-t / \tau) ; \text { with } \tau=\frac{C A}{P h} .
$$

From the experimentally obtained $\tau$ values, and using the measured $C(T)$ at the start of the temperature decay (see $C(T)$ data in table 1), we have estimated the values of $h$ for the samples S1 and S2. The results are plotted in figure 6.

For both analysed samples S1 and S2 we observed four cases:

i) Convective regime with $h=100-200 \mathrm{Wm}^{-2} \mathrm{~K}^{-1}$ and $\Delta T<2-5 \mathrm{~K}$ (squares in figure 6).

ii) Nucleate boiling regime characterized by much higher $h$ values (800-3000 $\mathrm{Wm}^{-2} \mathrm{~K}^{-1}$ ) when the temperature of the sample increases up to $\Delta T=5-10 \mathrm{~K}$ above liquid nitrogen temperature (circles in figure 6).

iii) Film boiling regime for $\Delta T=50-120 \mathrm{~K}$, characterized by low $h$ values of the order of $250-350 \mathrm{Wm}^{-2} \mathrm{~K}^{-1}$. These values correspond to the first time decay after the end of the pulse at the higher AC currents $\left(I_{\mathrm{p} \text {-short }}=450 \mathrm{~A}\right.$ in figure $3(d)$ and $270-307 \mathrm{~A}$ in figure 5). These data are represented by rhombus in figure 6.

iv) In the case of film boiling, when the temperature decreases again down to $\Delta T \sim 20-30 \mathrm{~K}$, there is a sudden increase in the rate of the temperature recovery. This temperature recovery is characterized in our case by $h$ values of the order of $2500-3500 \mathrm{Wm}^{-2} \mathrm{~K}^{-1}$ (triangles in figure 6).

The values obtained by Flynn et al [8] for a cylinder have also been plotted in figure 6(a) for comparison. Although taken into account the importance of the surface characteristics in these values, it must be noted the good correspondence between both set of data.

In the case of samples $\mathrm{S} 1$ and $\mathrm{S} 2$ at in the conditions of film boiling regime (iii), the $T(t)$ curves measured by the thermocouples have a maximum at the time $t \sim 0.12$ $0.20 \mathrm{~s}$, although the current had previously been switched off at $t \sim 0.09 \mathrm{~s}$ (see figures 4 and 5). This delay between the end of the current pulse and the maximum of $T(t)$ 
indicates the existence of either a thermal resistance between the thermocouple and the sample, or a temperature gradient between the sample surface and the rest of the tape.

In order to study this effect in more detail, and to verify the values measured by the thermocouples, we have estimated the temperature of the sample from the $V(t)$ measurements and the previously measured $\rho(T)$ curve. It must be noted that in this case, since the resistance is zero for $T<T_{\mathrm{c}}$, it is not possible to estimate temperatures that are smaller than $T_{\mathrm{c}}$ (i.e. $\Delta T<12 \mathrm{~K}$ ), as seen in figures 7 and 8 . The thermal recovery after the over-current pulse has been analysed in two cases, in which the current is set to zero and to an $\mathrm{AC}$ operating current, $I_{\mathrm{p} \text {-short }}=30 \mathrm{~A}$. The results are shown in figure 7 . In the case of recovery with an $\mathrm{AC}$ operating current, comparing the $\Delta T(t)$ curves measured by the thermocouple (dotted line) and estimated from the resistance (continuous line), the main difference is the lower temperature increase rate measured by the thermocouple during and just after the over-current pulse $(t<0.2 \mathrm{~s})$, while the rest of the curve is very similar. In fact, the $\tau$ values estimated in both cases at the first stages of the recovery, $t<0.6 \mathrm{~s}$, are very similar. $\tau=0.44 \mathrm{~s}$ is obtained from the $\Delta T(t)$ curves measured from the thermocouple while the resistivity curves lead to a value of $\tau=0.46 \mathrm{~s}$. It must be noted that the change of slope at $\Delta T \sim 30 \mathrm{~K}$ is observed in both cases. Moreover, since the resistance of the superconductor is high after the overcurrent pulse, the set operating current $\left(I_{\mathrm{p} \text {-short }}=30 \mathrm{~A}\right)$ is only achieved at $t \sim 0.7 \mathrm{~s}$ [see figure 7(a)], when the electric field tends to zero [figure 7(b)].

On the other hand, the difference between the $\Delta T(t)$ curves measured by the thermocouple in both cases of recovery with zero and non-zero current (dashed and dotted lines, respectively) is small. There is a small delay of $\sim 40 \mathrm{~ms}$ for complete recovery, and a small difference in $\tau$ value ( $\tau=0.35$ and $0.44 \mathrm{~s}$ for the cases of recovery without and with current, respectively, during the first stages of the recovery, $t<0.6 \mathrm{~s}$ ). It must be noted that the change of slope at $\Delta T \sim 30 \mathrm{~K}$ is observed in both cases.

In order to quantify the error caused by neglecting the longitudinal thermal conductivity term in equation (2) in the estimation of the heat transfer coefficient values, we have calculated numerically the $T(t)$ curves in two different conditions, in which $K=0$ and $K=K(T)$ estimated from the measured resistivity, $\rho(T)$, by the

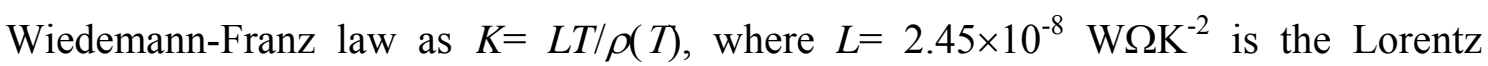
number, and using the experimental $\rho(T)$ and $C(T)$ curves. We have found that the difference between the recovery times in both cases is just of a few $\mathrm{ms}$, which indicates 
that the above approximation is valid in our case, and simplifies the estimation of the $h$ values of this system.

\subsection{Current limitation under fault currents}

Since the maximum current of the experimental set-up is about $450 \mathrm{~A}$, just about 3-3.5 times the critical current of these $4 \mathrm{~mm}$-wide tapes, it is not possible to analyse these coated conductors under fault currents of 5-10 times higher than the critical current. With this aim, we have analysed the effect of fault currents of up to $307 \mathrm{~A}$ on a $2 \mathrm{~mm}$-wide tape with $I_{\mathrm{c}}(77 \mathrm{~K})=40 \mathrm{~A}$ (sample S3). Figure 8 shows the current, electric field and temperature at the centre of tape S3 when a fault current of $I_{\mathrm{p} \text {-short }}=307 \mathrm{~A}$ is applied to the sample, followed by an operating current $I_{\mathrm{p} \text {-short }}=30 \mathrm{~A}$. The current in the circuit in this case is limited to $I_{\mathrm{p}} \sim 95 \mathrm{~A}$ at the beginning of the fault, which is 2.4 times of $I_{\mathrm{c}}$, and decreases down to $I_{\mathrm{p}} \sim 36 \mathrm{~A}$ after 4 cycles. Moreover, it is observed that the sample behaves very homogenously. The three electric fields measured between each two adjacent pair of the four voltage taps differ in less than a $10 \%$. Note that, as shown in figure 7 for sample $S 2$, the operating current $\left(I_{\mathrm{p} \text {-short }}=30 \mathrm{~A}\right)$ is limited during the thermal recovery of the sample. The recovery time, about $0.7 \mathrm{~s}$, and the shape of the time decay of the temperature recovery with time is very similar to that measured for the sample $\mathrm{S} 2$. There is a slow decrease of $\Delta T(t)$ down to $\sim 30 \mathrm{~K}$, followed by a sudden decrease of the recovery time constant (from $\tau \sim 0.4-0.5$ to $0.03 \mathrm{~s}$ ), very similar to the values obtained for sample S2 (see figures 6(b) and 7). In this case, there is also a difference in the temperature measured by the thermocouples at the tape surface and that estimated from the resistivity, during and just after the over-current pulse $(t<0.2 \mathrm{~s})$ as shown in figure $8(\mathrm{c})$.

Figure 9 shows the values of $I_{\mathrm{p}}$ as a function of $I_{\mathrm{p} \text {-short }}$ in order to analyse the limiting characteristics of these samples. It is observed that the sample with copper stabilizer (S1) limits the current just slightly at the end of the pulse. On the contrary, both non-stabilized samples limit the current considerably for $I_{\mathrm{p} \text {-short }}$ values higher than 1.8-2 times their $I_{\mathrm{c}}$. The value of $I_{\mathrm{p}}$ in this case is about 0.9 times $I_{\mathrm{c}}$ in both samples: 36 A and 126 A for samples S3 and S2, respectively. 


\section{Conclusions}

We have analysed the behaviour of $\mathrm{Cu}$-stabilized and non-stabilized YBCO coated conductors immersed in liquid nitrogen subject to $\mathrm{AC}$ and $\mathrm{DC}$ over-currents. It has been observed that samples with $\mathrm{Cu}$-stabilization are very stable against overcurrents. Analysing the recovery time of both types of coated conductors, we have obtained the values of the heat transfer coefficient, which are similar for both samples: $h \sim 100-200 \mathrm{Wm}^{-2} \mathrm{~K}^{-1}$ for the convective regime (at $\Delta T<5 \mathrm{~K}$ ), increasing by an order of magnitude to $h \sim 800-3000 \mathrm{Wm}^{-2} \mathrm{~K}^{-1}$ for the nucleate boiling regime at $\Delta T=5-10 \mathrm{~K}$.

For the samples without $\mathrm{Cu}$-stabilization, we found that $\mathrm{AC}$ currents higher than $I_{\mathrm{p} \text {-short }}=270 \mathrm{~A}\left(1.9\right.$ times of $\left.I_{\mathrm{c}}\right)$ increase the temperature of the sample by $150-200 \mathrm{~K}$ above $77 \mathrm{~K}$ and drive to the film boiling regime. On the contrary, for $\mathrm{Cu}$-stabilized tapes higher over-currents, $I_{\mathrm{p} \text {-short }}=450 \mathrm{~A}$ (3.7 times of $I_{\mathrm{c}}$ ), are needed to observe this regime. In both cases, this is characterized by $h \sim 250-350 \mathrm{Wm}^{-2} \mathrm{~K}^{-1}$.

The current limiting performance by the superconductor under these overcurrents or fault conditions is different for each sample. The limiting effect of the analysed $\mathrm{Cu}$-stabilized tapes is very small (just limited to $95 \%$ at the end of a $90 \mathrm{~ms}-$ pulse of $\left.I_{\mathrm{p} \text {-short }}=307 \mathrm{~A}=2.4 I_{\mathrm{c}}\right)$. In contrast, for the non-stabilized tapes, the current limiting effect is clearly observed: for prospective fault currents of $I_{\mathrm{p} \text {-short }}=307 \mathrm{~A}$ with $90 \mathrm{~ms}$ duration, the current was limited to $40 \%$ and $13 \%$ at the end of the pulse for tapes with $I_{\mathrm{c}}=140$ and 40 A, respectively.

\section{Acknowledgments}

This work was supported by the Spanish Ministry of Education and Science, MAT200806279-C03-01.

\section{References}

[1] Kwon N Y, Kim H S, Kim K L, Yim S W, Kim H-R, Hyun O-B, Kim H M and Lee H G, 2009 Supercond. Sci. Technol. 22045003

[2] Angurel LA, Amaveda H, Natividad E, Castro M, Andrés JM, Bona MT, 2007 IEEE Trans. Appl. Supercond. 173012

[3] Furuse M, Yamasaki H, Manabe T, Sohma M, Kondo W, Yamaguchi I, Kumagai T, Kaiho K, Arai K and Nakagawa M, 2007 IEEE Trans. Appl. Supercond. 173479 
[4] Kim H M, Jankowski J, Lee H, Bascuñán J, Fleshler S and Iwasa Y, 2004 IEEE Trans. Appl. Supercond. 141290

[5] C. Peroz C, Villard C, Buzon D and Tixador P 2003 Supercond. Sci. Technol. 1654

[6] Wang X, Trociewitz U P and Schwartz J 2007 J. Appl. Phys. 101053904

[7] Ishiyama A, Yanai M, Morisaki T, Ueda H, Shiohara Y, Izumi T, Iijima Y and Saitoh T, 2005 IEEE Trans. Appl. Supercond. 151659

[8] Flynn T M, Draper J W and Roos J J 1962 Adv. Cryog. Eng.7 539

[9] Sakurai A, Shiotsu M and Hat K, 1992 Cryogenics 32421

[10] Chovanec F and Usak P, 2005 Cryogenics 45129

[11] Baley W, Young E A, Yang Y and Beduz C 2006 Supercond. Sci. Technol. 19276 
Table 1. Parameters of the analysed conductors.

\begin{tabular}{cccc}
\hline Sample ID & $\mathrm{S} 1$ & $\mathrm{~S} 2$ & $\mathrm{~S} 3$ \\
\hline Stabilizer material & $\mathrm{Ag} / \mathrm{Cu}$ & $\mathrm{Ag}$ & $\mathrm{Ag}$ \\
Copper stabilizer thickness & $20+20 \mu \mathrm{m}$ (both sizes) & 0 & 0 \\
Silver thickness & $2+1 \mu \mathrm{m}$ (both sizes) & $2+1 \mu \mathrm{m}$ (both sizes) & $2+1 \mu \mathrm{m}$ (both sizes) \\
$\begin{array}{c}\text { Substrate thickness } \\
\text { (hastelloy) }\end{array}$ & $50 \mu \mathrm{m}$ & $50 \mu \mathrm{m}$ & $50 \mu \mathrm{m}$ \\
Total thickness & $95 \mu \mathrm{m}$ & $55 \mu \mathrm{m}$ & $55 \mu \mathrm{m}$ \\
Width, $w$ & $4 \mathrm{~mm}$ & $4 \mathrm{~mm}$ & $2 \mathrm{~mm}$ \\
Critical current at $77 \mathrm{~K}$ & $123 \mathrm{~A}$ & $140 \mathrm{~A}$ & $40 \mathrm{~A}$ \\
Heat capacity $\left(\mathrm{JK} \mathrm{cm}^{-3}\right)$ at & $1.56,2.07,2.76,3.08$, & $1.36,1.80,2.40,2.68$, & \\
$77,100,150,200,300 \mathrm{~K}$ & 3.37 & 2.81 & \\
Resistance/length $(\Omega / \mathrm{m})$ at & $0.019,0.036,0.069,0.085$ & $0.118,0.197,0.273,0.417$ & $0.487,0.757,1.009,1.483$ \\
$100,150,200,300 \mathrm{~K}$ & & & \\
\hline
\end{tabular}




\section{Figure Captions}

Figure 1. Electric field (a) and temperature increase (b) of sample S1 (Cu-stabilized) for DC current pulses of 140,145 and 147 A (corresponding to $I / I_{\mathrm{c}} \sim 1.14,1.18$, and 1.19 , respectively). The pulse started at $t=0 \mathrm{~s}$ and current reached a constant value at $t \sim 0.33 \mathrm{~s}$.

Figure 2. Electric field (a) and temperature increase (b) of sample S1 (Cu-stabilized) for AC current pulse of $I_{\mathrm{p} \text {-short }}=178 \mathrm{~A}\left(I_{\mathrm{p} \text {-short }} / I_{\mathrm{c}}=1.45\right)$ and duration $4 \mathrm{~s}$. The insets show a detail of $E(t)$ and $I(t)$ at the beginning of the pulse, and at around $t=3 \mathrm{~s}$ when the sudden decrease of $E(t)$ and $T(t)$ are observed.

Figure 3. Current (dotted lines) and electric fields (continuous lines) of sample S1 (Custabilized) for short AC current pulses of $I_{\mathrm{p}-\text { short }}=235 \mathrm{~A}$ (a), $307 \mathrm{~A}$ (b) and $450 \mathrm{~A}$ (c) ( $I_{\mathrm{p} \text { - }}$ short $/ I_{\mathrm{c}}=1.9,2.5$ and 3.7, respectively) and duration about $90 \mathrm{~ms}$. The three electric fields (measured between adjacent voltage taps), which are very similar, are shown. (d) Temperature increase at the sample surface, measured by the thermocouple at the centre on the sample.

Figure 4. Time dependence of the current and the electric field for the non-stabilized sample S2 subject to an AC over-current pulses of $I_{\mathrm{p} \text {-short }}=235 \mathrm{~A}$ (a) and $307 \mathrm{~A}$ (b) ( $I_{\mathrm{p} \text { - }}$ short $/ I_{\mathrm{c}}=1.7$ and 2.2 , respectively) and duration $\sim 90 \mathrm{~ms}$. The three measured electric fields (signals measured between adjacent voltage taps: $E_{12}, E_{23}$ and $\left.E_{34}\right)$ are shown.

Figure 5. Time dependence of $\Delta T$ measured by the thermocouple in the centre of the non-stabilized sample S2 in a log-scale, for different AC over-current pulses $\left(I_{p \text {-short }}=205\right.$, $235,265,270$ and $307 \mathrm{~A}$ ) of duration $\sim 90 \mathrm{~ms}$.

Figure 6. (a) Heat transfer coefficient, $h$, and (b) $\tau$ values for $\mathrm{Cu}$-stabilized sample $\mathrm{S} 1$ (closed symbols) and the non-stabilized tape S2 (open symbols) estimated from the temperature decays after short pulses ( $\sim 90 \mathrm{~ms})$ using equation (3). The squares correspond to the convective regime (i), the circles to nucleate boiling (ii), rhombus to film boiling (iii), and the triangles to the regime described in iv), (see text). The dotted line corresponds to the values obtained by Flynn et al. [6] for comparison. 
Figure 7. Time dependence of the current (a), electric field between voltage taps 2-3, $E_{23}$ (b), and temperature increase at the centre of the sample (c) for the non-stabilized tape S2 after an AC short-circuit current pulse $I_{\mathrm{p} \text {-short }}=307$ A during $90 \mathrm{~ms}$. The current is set to $I_{\mathrm{p} \text {-short }}=30 \mathrm{~A}$ after the pulse. In (c) the temperatures measured by the thermocouple (dotted line) and estimated from the $V_{23}(t)$ and $\rho(T)$ curves (continuous line) are shown. The recovery behaviour in which the current is set to zero after the pulse is also shown in (c) (dashed line) for comparison.

Figure 8. Time dependence of the current (a), electric field between voltage taps 2-3, $E_{23}$ (b) and temperature increase at the centre of the sample (c) of the non-stabilized tape S3 for a fault current $I_{\mathrm{p} \text {-short }}=307 \mathrm{~A}$ during $90 \mathrm{~ms}$. The current is set to $I_{\mathrm{p} \text {-short }}=30 \mathrm{~A}$ after the pulse. In (c) the temperature measured by the thermocouple (dotted line) and estimated from the resistance (continuous line) is shown. The recovery behaviour in which the current is set to zero after the pulse is also shown in (c) (dashed line) for comparison.

Figure 9. Current limitation of all the analysed samples $\mathrm{S} 1, \mathrm{~S} 2$ and $\mathrm{S} 3$ at the beginning (open symbols) and at the end of the AC over-current pulse (closed symbols). 

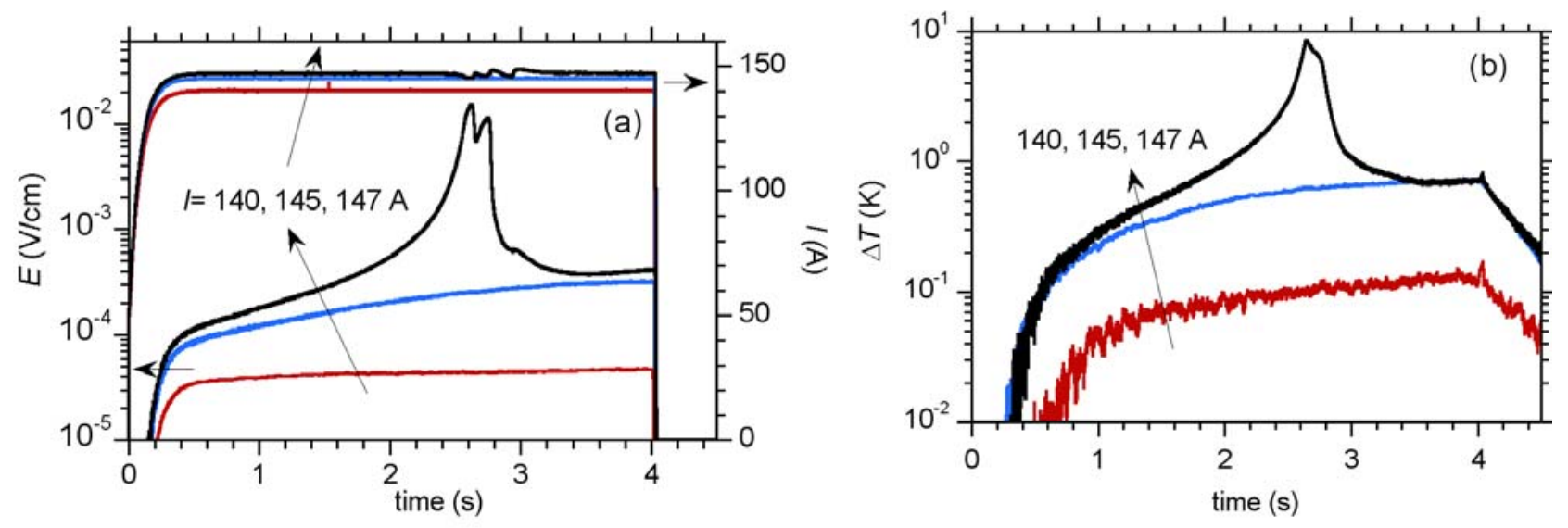

Figure 1. 

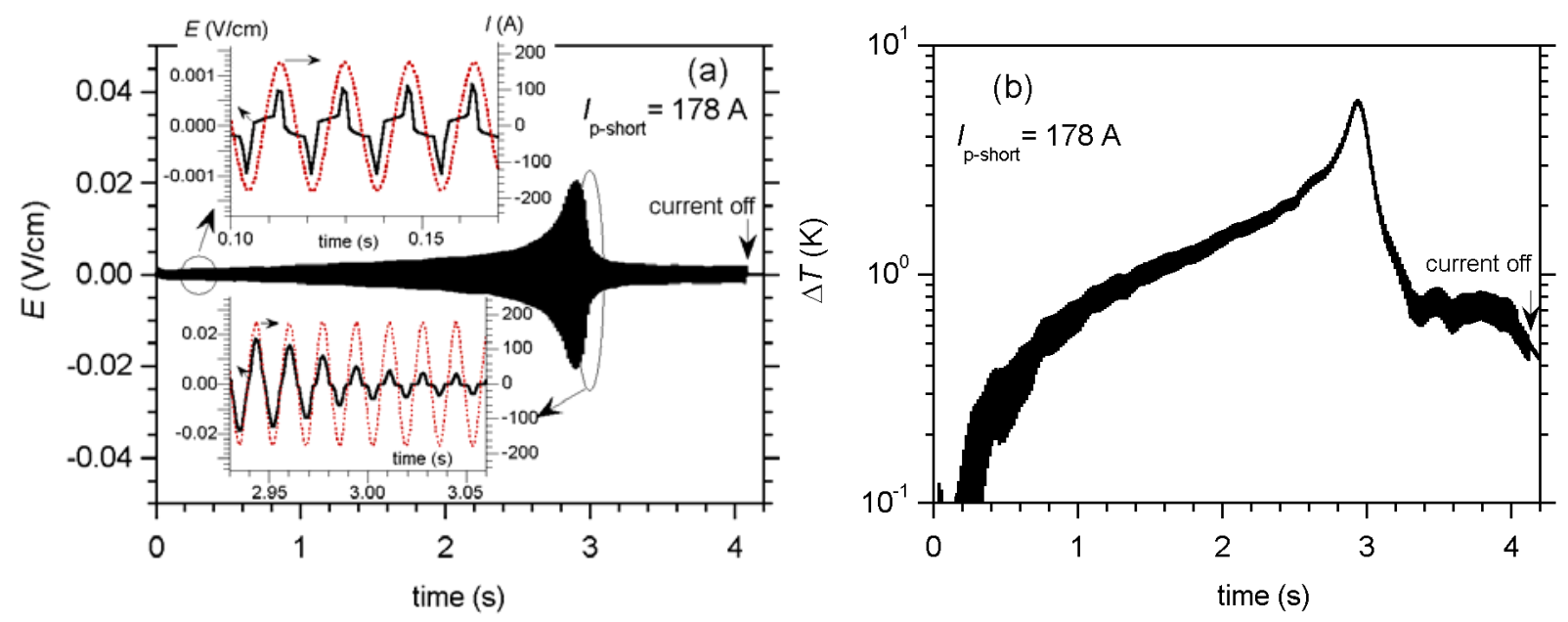

Figure 2. 

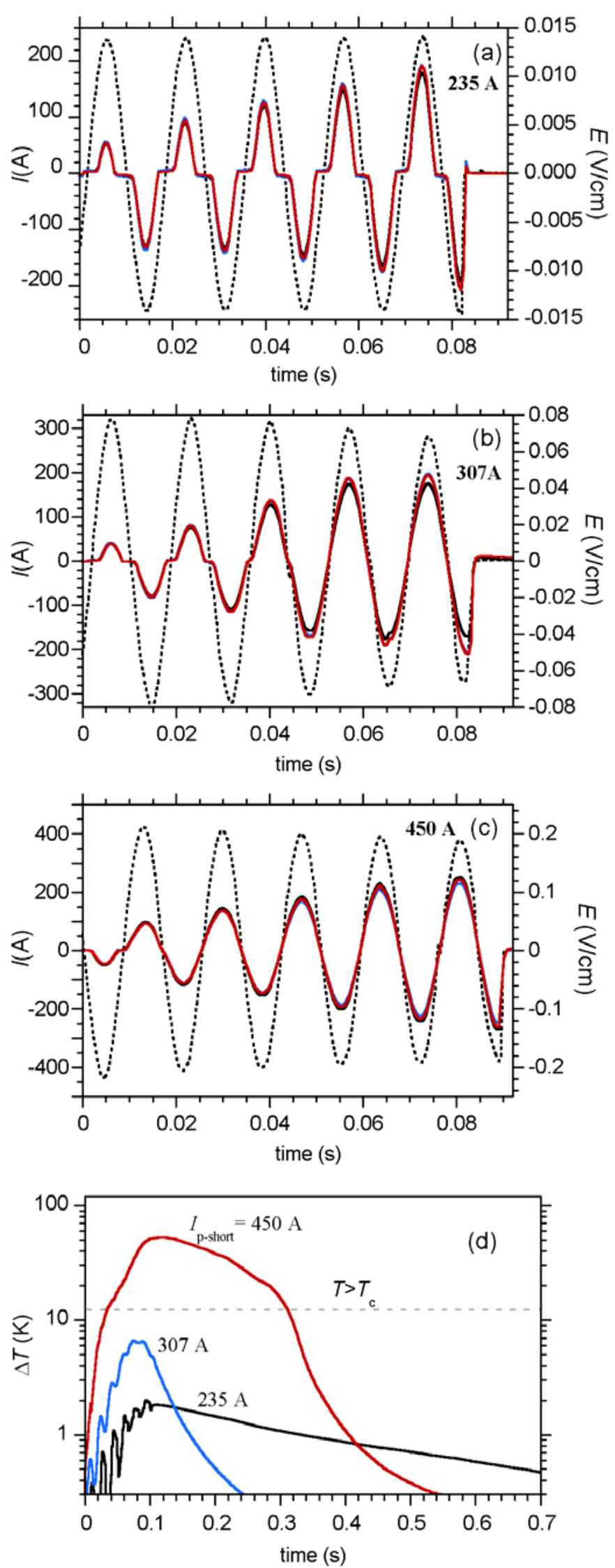

Figure 3. 


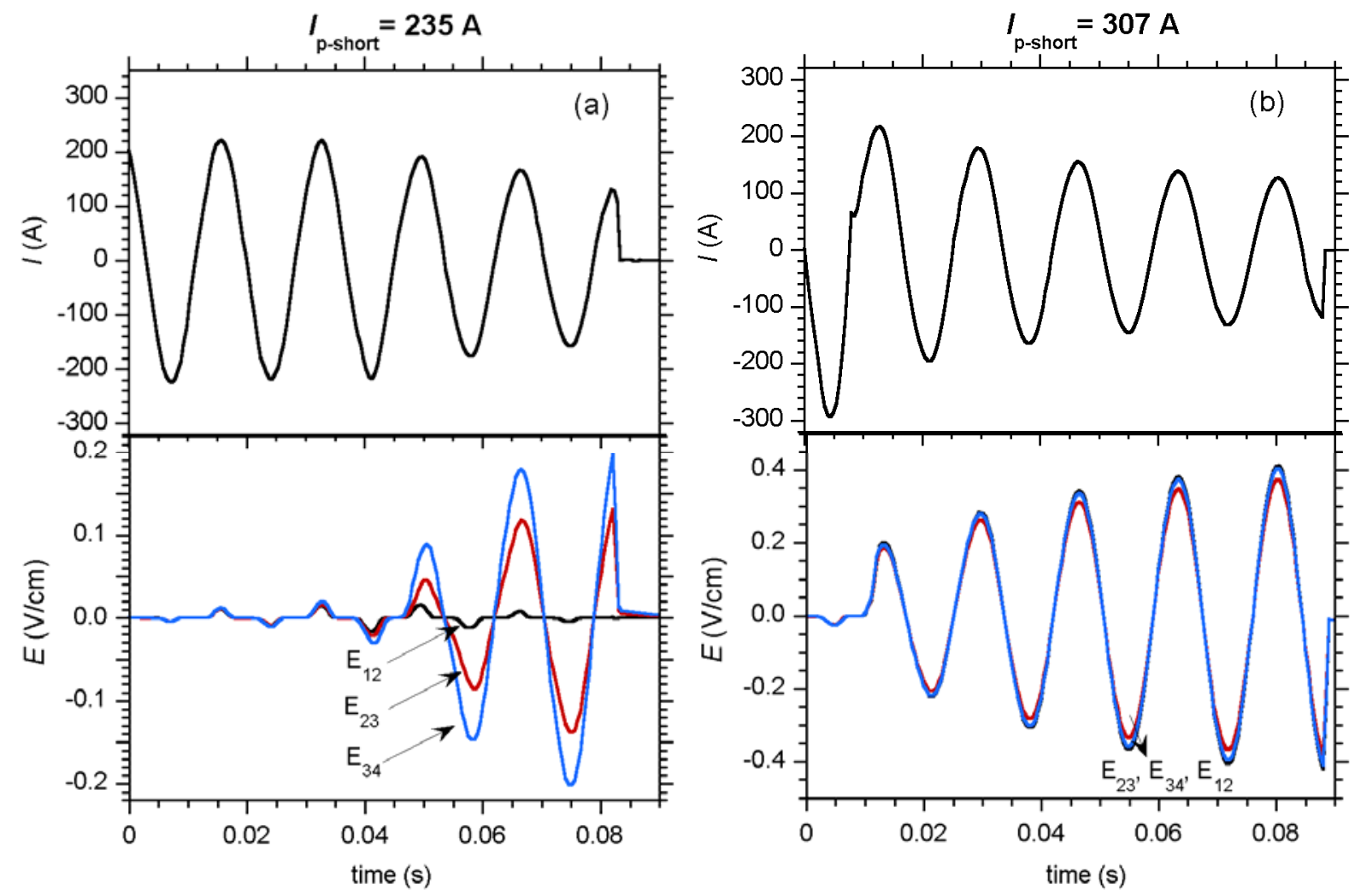

Figure 4. 


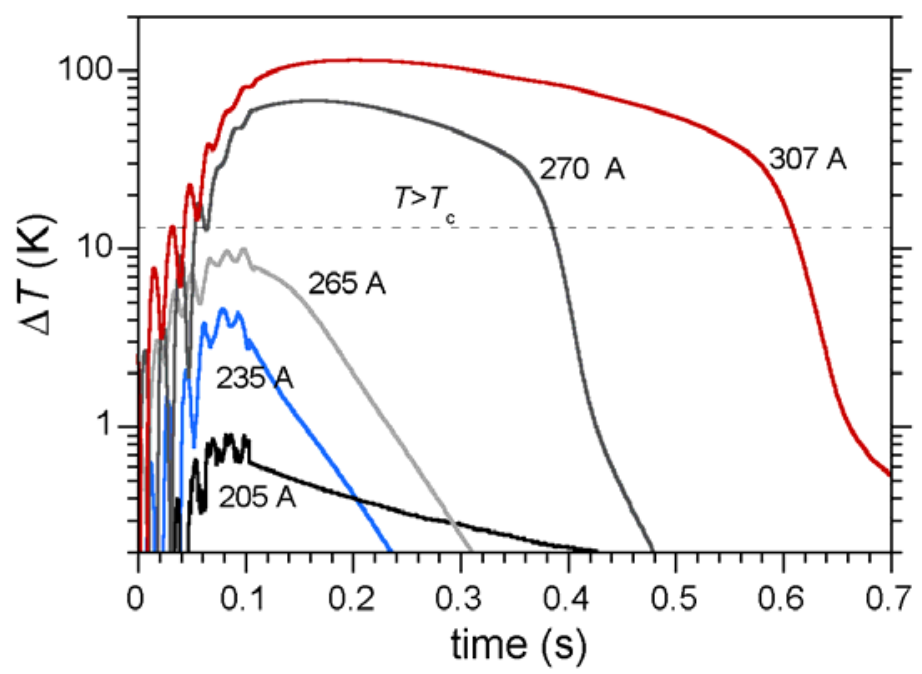

Figure 5. 


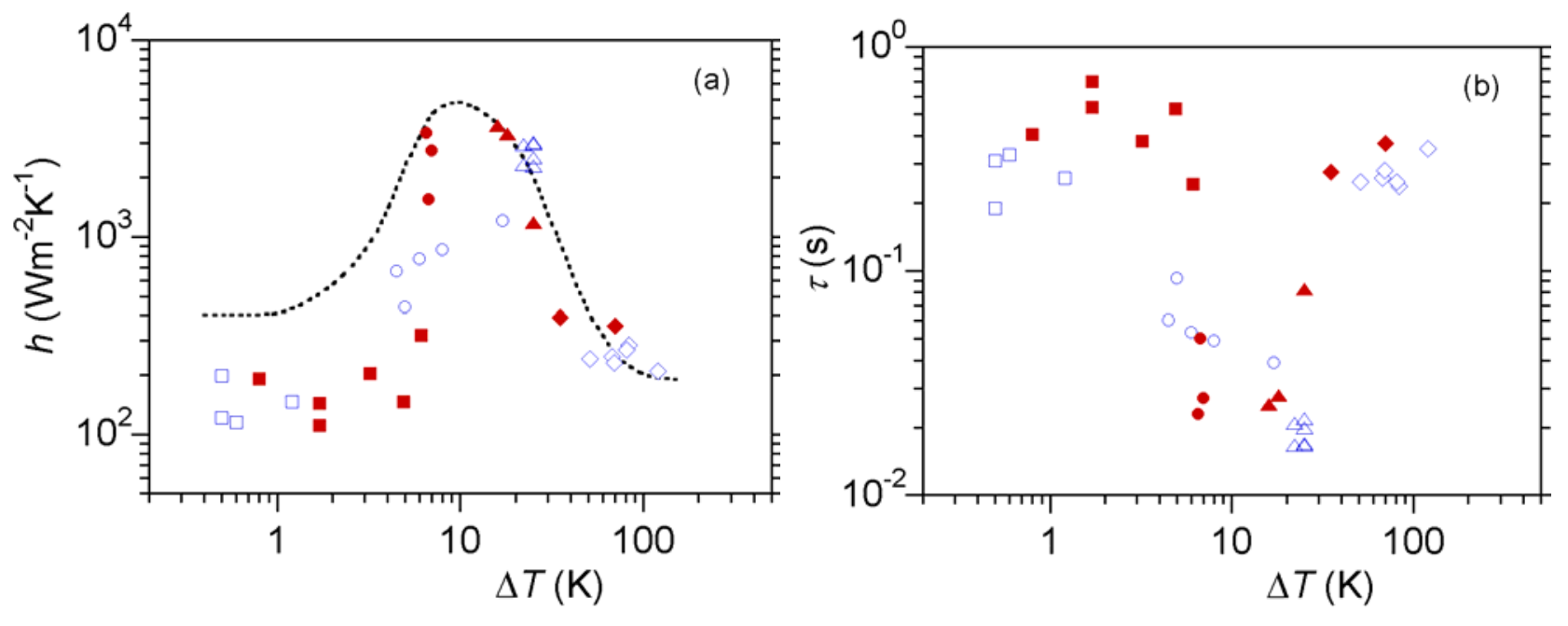

Figure 6. 


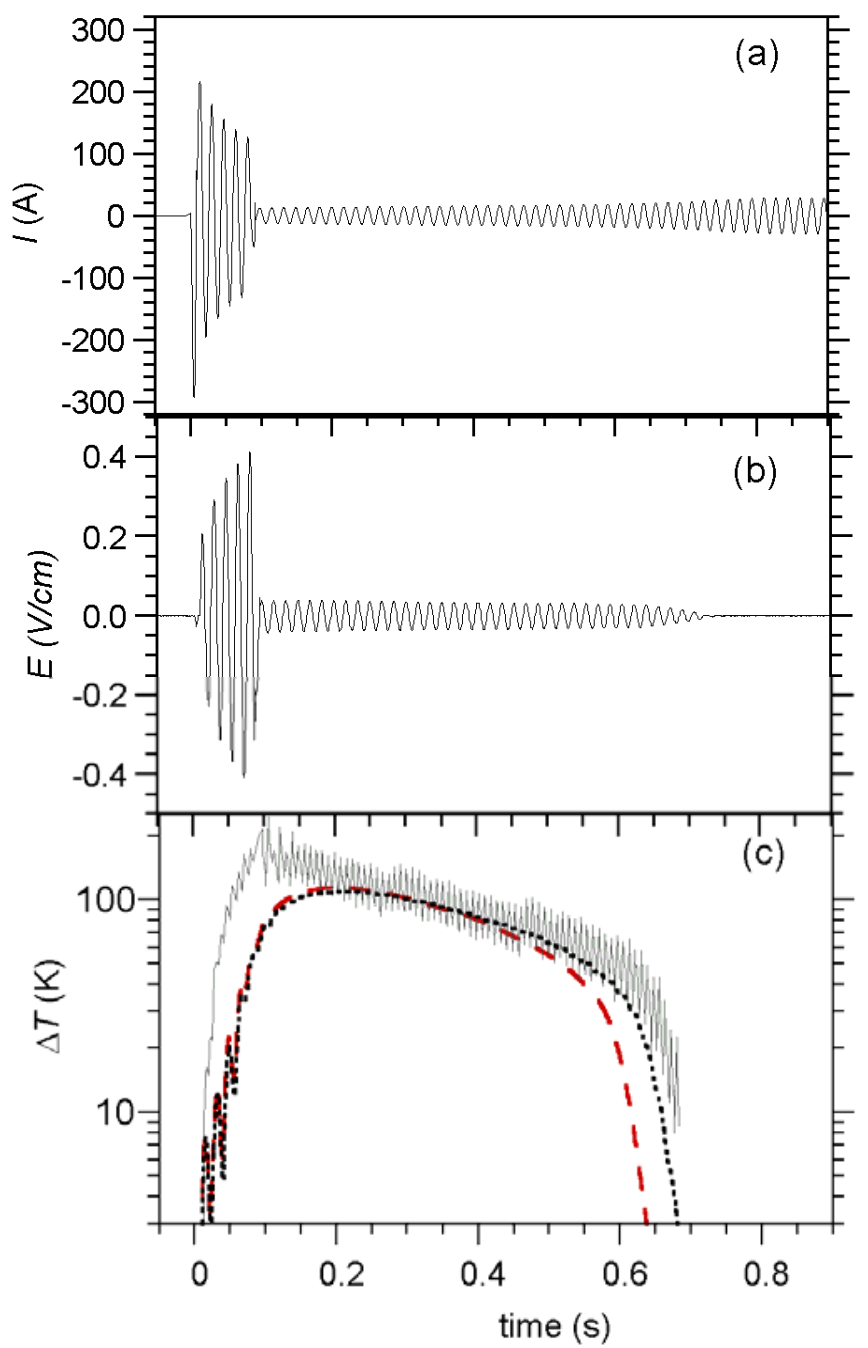

Figure 7 


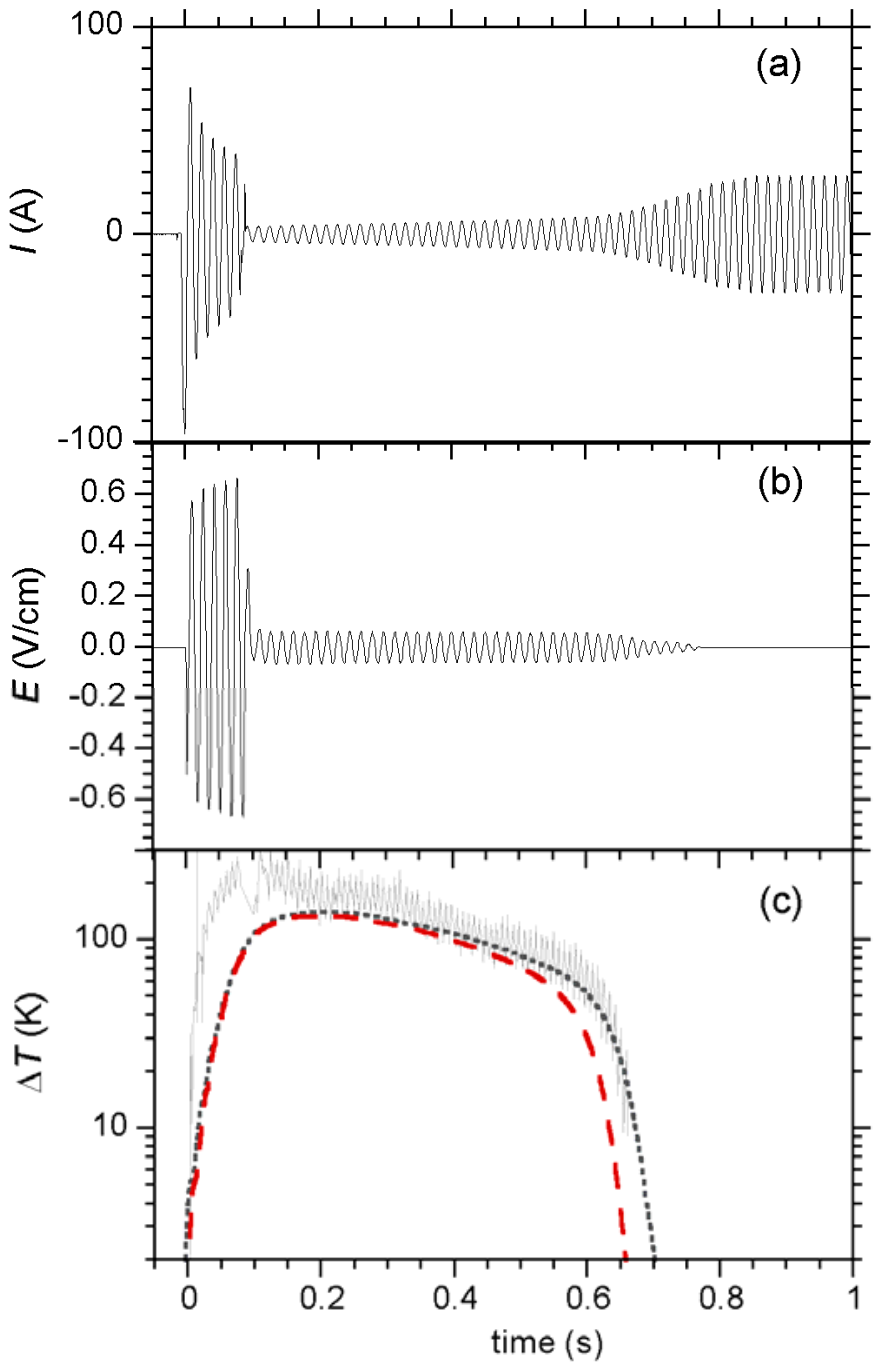

Figure 8. 


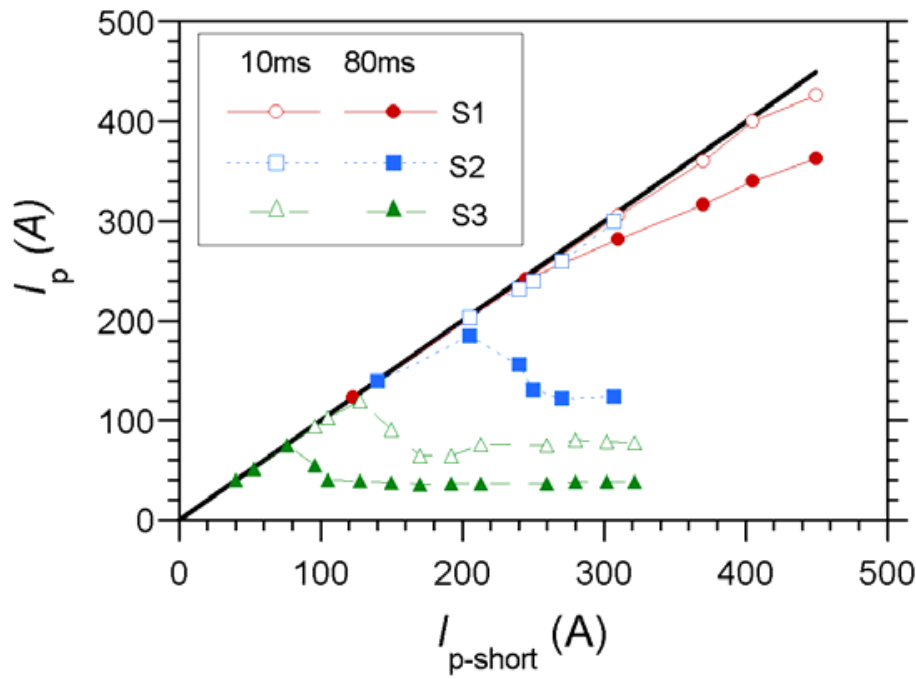

Figure 9. 